Session (Draft Doc. 2003-892)

\title{
Course Assessment Tools and Methods Utilizing Assignments, Tests and Exams
}

\author{
John R. Hackworth, Richard L. Jones \\ Old Dominion University
}

\section{Introduction}

Until recently, course assessment methods have been relegated to simply having an instructor examine results of assignments, tests and, exams, and making subjective determinations of how well the class is performing. This includes an "educated guess" as to whether or not students are grasping concepts being delivered in lecture classes (and supported in laboratory classes) and formulating an overall opinion of how the class is performing. When this method is used, in most cases, when queried about class performance, the instructor has little more to contribute than, They're doing ok," or "I think they're doing better than last semester's class," or "I believe this is the best class I've ever had," or some similar phrase. Seldom does the instructor have a quantitative value that can be assigned to the class performance, and rarely is he or she able to pinpoint which particular subjects within the course curriculum were problematic. The result is that the instructor usually lacks the data necessary to take the corrective action that can result in improvement in class (and individual student) performance.

In this paper, we will examine a method using assignments, test, and exams in a closed loop assessment system that will allow the instructor to better evaluate the performance of the curriculum, and determine specifically what changes need to be made to improve the quality of the course and the quality of the student who has completed the course. A complete methodology will be demonstrated which takes the reader from the early stages of course development through critical milestones. These include the development of course objectives; the mapping of assignments, tests, and exams to each of the course objectives; the extraction, compilation, and evaluation of resulting data; and the use of these data to provide continuous improvement in the course content and student evaluation methods. This method is independent of the course delivery method (live, TV broadcast, internet streamed, CD-ROM, etc.). Samples of an actual course assessment using this method is presented which show how the data is extracted, how it is related to the course objectives, and interpretations of the information contained in the data. 


\section{Assessment Methodology}

Effective course assessment is not a task that can be done using any "seat-of-the-pants" method, nor can it be initiated after a course is completed. To effectively assess a course, one must instead begin planning for assessment prior to the beginning of the course, and keep assessment in the forefront of the planning throughout the course.

Assessment planning and execution can be divided into several unique but integrated tasks. These are a) determine the course objectives, b) map graded assignment, test, and exam questions and problems to the course objectives, c) compile the data, d) make adjustments.

\section{a) Course Objectives}

Selecting the course objectives is the most critical step in the assessment process. These are not only the list of learning goals for the class, but they are also the goals to which every graded item will be mapped and compared. It is good practice to make the objectives match major milestones within the course. Since most courses use textbook chapters as "stepping stones", a logical practice is to create one objective for each text chapter, as shown in Figure 1a. In some cases, this could result in more than one learning goal being included in a single assessment objective. The result of this process should be typically 8-16 objectives.

a)

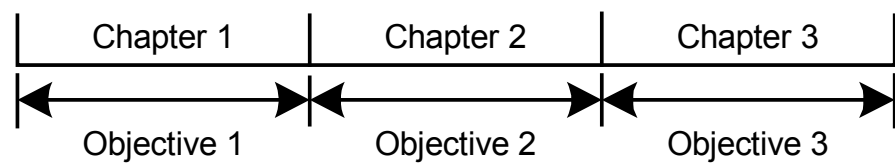

b)

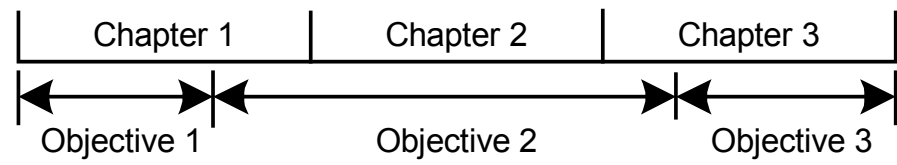

Figure 1 - Objective-to-Chapter Mapping, a) Direct Mapping, and b) Shared/Spanned Mapping
The reader is cautioned against creating an objective that both shares a chapter with another objective, and also spans more than one chapter, as shown in Figure $1 \mathrm{~b}$. The reason is that, since the problems in graded assignments are generally grouped by chapters, the instructor will then find it necessary to map an assignment to multiple objectives, which is a rather tedious process

that must be done while grading the assignment. The assessment process is much simpler if each entire graded assignment can be mapped to a single objective.

Number the objectives. If one objective is assigned to each chapter of the textbook, number the objectives the same as the chapter numbers. If it is desirable to have more than one objective mapped to a single chapter in the text, it is helpful to number each of them with the same chapter number, but with a letter delineating them (e.g., for chapter 3, Objectives $3 \mathrm{a}$ and $3 \mathrm{~b}$ ). In the end, the selection of a simple and logical course objective numbering scheme will pay off by simplifying the assessment process, and can eliminate then need for a cross reference that relates objectives to chapters or subjects.

For each objective, determine a numerical value that is considered to be acceptable. That is, what is the minimum numerical score that the class can obtain on each objective that shows

"Proceedings of the 2003 American Society for Engineering Education Annual Conference \& Exposition Copyright (C) 2003, American Society for Engineering Education" 
that the class has attained the particular goal? In this step, it is entirely possible that every objective can have a different minimum score. For example, if one objective is relatively easy subject material, then one would expect the class to score higher on it than they would on more difficult subjects.

b) Map questions and problems on graded assignments, tests, and exams to the course objectives

With this type of assessment method, all of the collected data will be extracted from graded assignments, tests, and exams. Therefore, the selection of problems for the graded assignments, and the formulation of questions and problems for tests and exams will play a key role in the quality of the data collected, and the ease with which it is collected.

For graded assignments, it is best to avoid bridging an assignment over several course objectives, because doing so will require that the problems on the assignment be individually mapped to respective objectives. Instead, if the entire assignment targets a single objective, then the class average grade on that particular assignment can be easily mapped in its entirety to one objective. In an effort to make data collection simpler, giving more frequent assignments with fewer problems (where all problems on each assignment relate to one objective) is preferable to fewer large assignments that span several objectives (that must be broken down problem by problem).

For tests and exams, there should be a collection of questions and problems that evaluate knowledge and skills relating to each objective. It is preferable to have an equal number of questions and problems assigned to each objective so that the weighting is evenly distributed. Try to avoid chain problems (where the answer to one problem is used as and input to the next problem) which can skew results. As with graded assignments, compilation of data is made easier by grouping together questions and problems related to a single objective. However, if it is desired to randomly mix subjects on a test or exam, the sorting and mapping can be accomplished using a spreadsheet, although this will obviously require more instructor time and effort.

c) Compile the data

At the end of the course, setup a spreadsheet with one row for each of the objectives. In the columns, list the graded assignments (one assignment per column), and the groups of problems on tests and exams. Then map each assignment, or test and exam group, to each objective by entering the class average score in the appropriate cell in the spreadsheet.

For each row, calculate the average score for each objective. When performing this operation, it may be desirable to weight entries. For example, if an instructor allows students to freely collaborate on assignments, it may be desired that graded assignments should contribute a lower percentage to the overall average of the objective.

d) Make adjustments (closing the loop)

The phrase "making adjustments" not only refers to adjustments in the course curriculum, but also adjustments in the assessment process itself. Often this process uncovers problems in

"Proceedings of the 2003 American Society for Engineering Education Annual Conference \& Exposition Copyright (C) 2003, American Society for Engineering Education" 
assignments and test that would not otherwise be noticed. For example, this assessment method will help in locating a poorly worded problem on an exam, or a multiple choice question with more than one possible correct answer.

Additionally, this assessment process may uncover that students are performing poorly in a complex concept because they did not adequately grasp one of the fundamental concepts covered earlier in the course. For example, in a DC electrical circuits course, a low score on an objective related to circuit reduction could be caused by lack of a fundamental concept such as Thevenin's and Norton's Theorems.

\section{Assessment Example}

As an example of how this assessment process is executed, we will examine an actual course assessment used at Old Dominion University in the course EET415 Programmable Machine Controls. It was decided for this particular semester that the assessment would be based on the homework assignments and the final exam. Although three intermediate tests were given during this course, it was decided at the outset not to use this data. In hindsight, this additional data would have been helpful as will be seen in the evaluation of the results. Also, as will be shown, additional planning is needed in the next offering of this course in order to collect additional data from graded homework assignments.

We began by defining the course objectives. The objectives related directly to the textbook chapters (one objective per chapter).

EET415 Objectives: Upon successful completion of this course you will...

1. ... know the electrical schematic symbols used in machine control diagrams and the special way these schematics are drawn.

2. ... know the fundamentals of how a PLC operates, and be able to specify and select a PLC and its discrete input/output modules.

3. $\quad . .$. be able to perform fundamental programming of a PLC using ladder logic.

4. $\quad . .$. know how to program advanced functions in a PLC.

5. $\quad . .$. be able to program a PLC using mnemonic code.

6. $\quad . .$. know how to wire a complete PLC system.

7. $\quad . .$. understand how analog I/O operates and be able to select analog I/O for a PLC.

8. ... know how various types of discrete position sensors operate, and be able to select the best sensor for an application.

9. $\quad . .$. understand and apply encoders, transducers and advanced position sensor.

10. ... know how a PID control system works and be able to tune a PID.

11. ... have a fundamental understanding of DC and AC motor controls and how to interface a motor control to a PLC.

12. ... understand industrial safety as related to machine controls.

The graded homework for this course consisted of seven assignments covering electrical controls design, schematics, and PLC programming. The assignments related to the first six chapters of the text, which are also the first six objectives.

"Proceedings of the 2003 American Society for Engineering Education Annual Conference \& Exposition Copyright (C) 2003, American Society for Engineering Education" 
The mapping of these assignments to their objectives, and the overall class scores are shown in Table 1. Note that there are no graded homework assignments that map to objectives 2 , and 7 through 12 . The reason for this is that chapters (and objectives) 2, and 7 through 12 related to informational topics. Instead, the graded assignments for this course targeted PLC programming techniques, which are subjects covered in chapters 1 and 3 through 6 of the text.

From a quick examination of the results in Table 1, it is appropriate to conclude that, based on graded homework

\begin{tabular}{|r|r|r|}
\hline $\begin{array}{l}\text { Assignment } \\
\text { Number }\end{array}$ & $\begin{array}{l}\text { Objective } \\
\text { Number }\end{array}$ & $\begin{array}{l}\text { Class } \\
\text { Avg }\end{array}$ \\
\hline 1 & 1 & 88.6 \\
\hline 2 & 1 & 80.7 \\
\hline 3 & 3 & 94.3 \\
\hline 4 & 4 & 85.5 \\
\hline 5 & 5 & 80.7 \\
\hline 6 & 5 & 83.6 \\
\hline 7 & 6 & 84.7 \\
\hline
\end{tabular}

Table 1 - Graded Assignment Results assignments only, the class is performing adequately on course objectives 1, and 3 through 6 .

The final exam was constructed with five questions/problems related to each objective. The question were grouped so that questions 1 through 5 mapped to objective 1, questions 6 through 10 mapped to objective 2, and so forth, with questions 55 through 60 mapped to objective 12 .

The results of each question on the exam were compiled to obtain an average score on each question. Table 2 shows each of the average scores, and the mapping of the questions on the exam to each of the objectives. In this table, the headings Q1 through Q5 correspond to the $\mathrm{n}^{\text {th }}$ question related to each particular objective. Question Q5 of objective 5 is missing data because the exam question was thrown out due to a wording error.

Based on the data in Table 2, several conclusions can be drawn that can be used for course improvement.

\begin{tabular}{|r|r|r|r|r|r|r|}
\hline Objective & Q1 & Q2 & Q3 & Q4 & Q5 & Avg \\
\hline 1 & 97 & 85 & 86 & 26 & 94 & 77.6 \\
\hline 2 & 77 & 94 & 88 & 94 & 88 & 88.2 \\
\hline 3 & 92 & 85 & 89 & 74 & 98 & 87.6 \\
\hline 4 & 74 & 35 & 91 & 36 & 77 & 62.6 \\
\hline 5 & 71 & 89 & 92 & 73 & & 81.3 \\
\hline 6 & 74 & 86 & 68 & 94 & 82 & 80.8 \\
\hline 7 & 91 & 92 & 45 & 65 & 14 & 61.4 \\
\hline 8 & 83 & 67 & 89 & 89 & 39 & 73.4 \\
\hline 9 & 65 & 94 & 100 & 91 & 67 & 83.4 \\
\hline 10 & 97 & 67 & 53 & 65 & 58 & 68.0 \\
\hline 11 & 94 & 92 & 73 & 62 & 94 & 83.0 \\
\hline 12 & 98 & 79 & 70 & 55 & 95 & 79.4 \\
\hline & & & & & & 77.2 \\
\hline
\end{tabular}

Table 2 - Exam Results

1. If we use a score of 70 as a breakpoint, the class met all objectives except for 4, 7, and 10 .

2. The low scores on objectives 4 and 7 appear to be exam related. There are apparent problems with question Q4 in objective 1, questions Q2 and Q4 in objective 4, questions Q3 and Q5 in objective 7, and question Q5 in objective 8. Possible reasons include unclear wording of the question, incorrect answer key, or more than one possible correct answer in a multiple choice question.

3. There is a problem with objective 10 that apparently is not exam related because the class scored low on 4 of the 5 questions. Although it needs further investigation, it appears the class did not meet course objective 10. The objective 10 subject material is PID control systems, one of the more difficult concepts covered in the course. This could relate to 
needed improvements in the text, method of instruction, presentation techniques, or even course prerequisites.

Additionally, one important observation was made during the final exam. Although three hours was allocated for the final exam, students finished the exam in an average of 90 minutes. Therefore, there is room to expand the exam to do a better job of collecting data, which includes more questions per objective and more in-depth questions.

\section{Summary}

A method using assignments, test, and exams in a closed loop assessment system has been shown that allows the instructor to better evaluate the performance of the curriculum, and determine specifically what changes need to be made to improve the quality of the course and the quality of the student who has completed the course. A complete methodology was demonstrated which took the reader from the early stages of course development through critical milestones.

\section{Bibliography}

1. Angelo, T. A., and K. P. Cross, 1993. "Classroom Assessment Techniques, A Handbook for College Teachers" (2nd Ed.) San Francisco: Jossey-Bass.

2. J. R. Hackworth, "A Video-Taped Laboratory in Electrical Power and Machinery. ASEE 2001 Annual Conference Proceedings.

\section{JOHN R HACKWORTH}

John R. Hackworth is Program Director for the Electrical Engineering Technology program at Old Dominion University. He holds a B. S. Degree in Electrical Engineering Technology and a Master of Science Degree in Electrical Engineering, both from Old Dominion University. Prior to joining the Old Dominion University faculty, John had approximately 20 years of industrial experience in test engineering and plant automation.

\section{RICHARD L. JONES}

Richard Jones has been teaching at ODU since 1994. He is a retired United States Navy Submarine Service Lt. Commander with sub-specialties in Ballistic Missile, Torpedo, Sonar, and Radio systems. Richard has previously taught Mechanical Engineering Design at the United States Military Academy (ARMY), West Point, N.Y., and Electrical Engineering at the United States Naval Academy, Annapolis, Md. He holds an ASEET from Cameron University, a BSEET from Oklahoma State University, and a Master of Engineering in Electronics Engineering from the Naval Postgraduate School at Monterey, California.

"Proceedings of the 2003 American Society for Engineering Education Annual Conference \& Exposition Copyright (C) 2003, American Society for Engineering Education" 